\title{
General decay for a viscoelastic wave equation with strong time-dependent delay
}

Baowei Feng ${ }^{*}$

\section{"Correspondence:}

bwfeng@swufe.edu.cn

Department of Economic

Mathematics, Southwestern

University of Finance and

Economics, Chengdu, 611130,

P.R. China

\begin{abstract}
A viscoelastic wave equation with strong damping and strong time-dependent delay in the internal feedback is considered. Under the assumption $\left|\mu_{2}\right|<\sqrt{1-d} \mu_{1}$, we establish the general decay of energy of the problem by using the energy perturbation method.
\end{abstract}

MSC: 35B35; 35B40; 93D15

Keywords: general decay; wave equation; memory; delay feedbacks

\section{Introduction}

In this paper, we consider the following viscoelastic wave equation with a strong damping and a strong time-dependent delay in the internal feedback:

$$
u_{t t}(x, t)-\Delta u(x, t)+\int_{0}^{t} g(t-s) \Delta u(s) d s-\mu_{1} \Delta u_{t}(x, t)-\mu_{2} \Delta u_{t}(x, t-\tau(t))=0
$$

where $x \in \Omega$, and $\Omega \subseteq \mathbb{R}^{n}(n \geq 1)$ is a bounded domain with smooth boundary $\partial \Omega$. The function $g(t)$ is the relaxation function. $\mu_{1}, \mu_{2}$ are constants and $\tau(t)>0$ denotes the timedependent delay.

We consider the following initial conditions:

$$
\left\{\begin{array}{l}
u(x, 0)=u_{0}(x), \quad u_{t}(x, 0)=u_{1}(x), \quad x \in \Omega \\
u_{t}(x, t)=f_{0}(x, t), \quad x \in \Omega, t \in[-\tau(0), 0)
\end{array}\right.
$$

and the following boundary conditions:

$$
u=0 \quad \text { on } \partial \Omega \times \mathbb{R}^{+} .
$$

Recently the control of wave equations with time delay effects has become an active area of research. The delay effects often appear in many practical problems and may turn a well-behaved system into a wild one. The presence of delay can be a source of instability. Here we mention the work of Nicaise and Pignotti [1]. In this work the authors considered a wave equation with time delay of the form

$$
u_{t t}-\Delta u+\mu_{1} u_{t}+\mu_{2} u_{t}(t-\tau)=0
$$

(c) The Author(s) 2017. This article is distributed under the terms of the Creative Commons Attribution 4.0 International License (http://creativecommons.org/licenses/by/4.0/), which permits unrestricted use, distribution, and reproduction in any medium, provided you give appropriate credit to the original author(s) and the source, provide a link to the Creative Commons license, and indicate if changes were made. 
Under the assumption $0<\mu_{2}<\mu_{1}$, they proved exponential stability of the system. For viscoelastic wave equation with time delay, Kirane and Said-Houari [2] studied the following equation:

$$
u_{t t}-\Delta u+\int_{0}^{t} g(t-s) \Delta u(s) d s+\mu_{1} u_{t}+\mu_{2} u_{t}(t-\tau)=0,
$$

and obtained the global well-posedness of solutions and established the energy decay under the assumption $0<\mu_{2} \leq \mu_{1}$. Liu [3] considered a wave with time-dependent delay

$$
u_{t t}-\Delta u+\int_{0}^{t} g(t-s) \Delta u(s) d s+\mu_{1} u_{t}+\mu_{2} u_{t}(t-\tau(t))=0,
$$

and established the general decay of the system under $\left|\mu_{1}\right|<\sqrt{1-d} \mu_{1}$. Dai and Yang [4] considered the same equation as in [2] and improved the above results under weaker conditions. In this work, the authors obtained the global well-posedness without any restrictions on $\mu_{1}, \mu_{2}$, and established an exponential decay result of energy in the case $\mu_{1}=0$. Benaissa et al. [5] investigated a wave equation with nonlinear time delay of the form

$$
u_{t t}-\Delta u+\int_{0}^{t} h(t-s) \Delta u(s) d s+\mu_{1} g_{1}\left(u_{t}(t)\right)+\mu_{2} g_{2}\left(u_{t}(t-\tau)\right)=0 .
$$

They proved the global existence of solution under assumption of a relation between the weight of the delay term in the feedback and the weight of the term without delay. In addition, they obtained the general decay of energy. Liu and Zhang [6] considered a wave equation with past history and time delay in internal feedback

$$
u_{t t}-\alpha \Delta u+\int_{-\infty}^{t} \mu(t-s) \Delta u(s) d s+\mu_{1} u_{t}+\mu_{2} u_{t}(t-\tau)+f(u)=h .
$$

They proved the global well-posedness without any restrictions on $\mu_{1}, \mu_{2}$. Furthermore, they also proved the exponential decay of energy with $0<\left|\mu_{2}\right|<\mu_{1}$. Kafini et al. [7] consider a nonlinear damped second-order evolution equation with delay

$$
u_{t t}+A u+G\left(u_{t}\right)+\mu G\left(u_{t}(t-\tau)\right)=F(u)
$$

and they proved that the energy of the solutions blows up in finite time under some suitable assumptions. Recently, Alabau-Boussouira et al. [8] studied a wave equation with past history and time delay

$$
u_{t t}-\Delta u+\int_{0}^{\infty} \mu(s) \Delta u(t-s) d s+k u_{t}(t-\tau)=0
$$

They showed that the system is exponentially stable if the coefficient of delay $k$ is small enough. They also established the stability in the case $\tau=0$ and $k<0$. For some more results concerning the wave equation with a weak time delay term under an appropriate assumption between $\mu_{1}$ and $\mu_{2}$, one can refer to Benaissa et al. [9], Datko et al. [10], Liu [11], Nicaise and Pignotti [12-14], Nicaise et al. [15], Nicaise and Valein [16], Xu et al. [17], and the references therein. With respect to waves with strong time delay there is just little 
published work. The only one we found is duo to Messaoudi et al. [18]. In this work, they investigated the following equation:

$$
u_{t t}-\Delta u-\mu_{1} \Delta u_{t}-\mu_{2} \Delta u_{t}(t-\tau)=0,
$$

and proved the well-posedness under the assumption $\left|\mu_{2}\right| \leq \mu_{1}$ and established exponential decay of energy under the assumption $\left|\mu_{2}\right|<\mu_{1}$. Moreover, they also studied a wave equation with distributed delay.

In the absence of time delay, problems similar to (1.1) have been extensively studied and there are many results in the literature, most of which are mainly concerned with global well-posedness, asymptotic behavior and blow-up. See, for example, Berrimi and Messaoudi [19], Cavalcanti et al. [20, 21], Han and Wang [22, 23], Liu [24], Messaoudi et al. [25-31], Tatar [32].

Motivated by [18], we study in the present work the asymptotic behavior for system (1.1)(1.3). Since the delay is dependent on time, this makes the work different from [18]. The main objective of the present work is to establish a general decay result from which the exponential decay and polynomial decay are only special cases.

The plan of this paper is as follows. In Section 2, we give some assumptions and our main results. In Section 3, we establish the general decay result of the energy by using the energy perturbation method.

\section{Assumptions and main results}

$L^{q}(\Omega)(1 \leq q \leq \infty)$ and $H^{1}(\Omega)$ denote the Lebesgue integral and Sobolev spaces. $\|\cdot\|_{B}$ is the norm in the space $B$, we write $\|\cdot\|$ instead of $\|\cdot\|_{2}$ for $q=2$.

For the relaxation function $g$, we assume $g: \mathbb{R}^{+} \rightarrow \mathbb{R}^{+}$is a nonincreasing $C^{1}$ function satisfying

$$
g(0)>0, \quad 1-\int_{0}^{\infty} g(s) d s=l>0,
$$

and there exists a nonincreasing differentiable function $\zeta: \mathbb{R}^{+} \rightarrow \mathbb{R}^{+}$satisfying, for $t \geq 0$,

$$
\zeta(t)>0, \quad g^{\prime}(t) \leq-\zeta(t) g(t)
$$

and

$$
\int_{0}^{\infty} \zeta(t) d t=\infty
$$

Concerning the delay $\tau(t)$, we assume

$$
0<\tau_{0} \leq \tau(t) \leq \tau_{1}, \quad \forall t>0
$$

where the constants $\tau_{0}$ and $\tau_{1}$ are two positive constants. We assume further that

$$
\tau(t) \in W^{2, \infty}(0, T) \quad \text { and } \quad \tau^{\prime}(t) \leq d<1, \quad \forall T, t>0 .
$$


The weak solutions of (1.1)-(1.3) are defined as follows: for given initial data $\left(u_{0}, u_{1}\right) \in$ $H_{0}^{1}(\Omega) \times L^{2}(\Omega)$, we call a function $U=\left(u, u_{t}\right) \in C\left(\mathbb{R}^{+}, H_{0}^{1}(\Omega) \times L^{2}(\Omega)\right)$ a weak solution to the problem (1.1)-(1.3) if $U(0)=\left(u_{0}, u_{1}\right)$ and

$$
\begin{gathered}
\left(u_{t t}, \omega\right)+(\nabla u, \nabla \omega)+\int_{0}^{t} g(t-s)(\Delta u(s), \omega) d s \\
+\mu_{1}\left(\nabla u_{t}, \nabla \omega\right)+\mu_{2}\left(\nabla u_{t}(t-\tau(t)), \nabla \omega\right)=0,
\end{gathered}
$$

for all $\omega \in H_{0}^{1}(\Omega)$.

The global well-posedness of problem (1.1)-(1.3) will be given in the following theorem.

Theorem 2.1 Let $\mu_{2} \leq \mu_{1}$, and assume the assumptions (2.1)-(2.4) hold. If the initial data $\left(u_{0}, u_{1}\right) \in\left(H_{0}^{1}(\Omega) \times L^{2}(\Omega)\right), f_{0} \in H^{1}(\Omega \times(-\tau(0), 0))$, then problem (1.1)-(1.3) has a unique weak solution $\left(u, u_{t}\right) \in C\left(0, T ; H_{0}^{1}(\Omega) \times L^{2}(\Omega)\right)$ such that, for any $T>0$,

$$
u \in L^{\infty}\left(0, T ; H_{0}^{1}(\Omega)\right), \quad u_{t} \in L^{\infty}\left(0, T ; L^{2}(\Omega)\right)
$$

Remark 2.1 By using the classical Faedo-Galerkin method, see, e.g., [2], we can prove the theorem and we omit the proof here.

The energy functional of problem (1.1)-(1.3) is defined by

$$
\begin{aligned}
E(t)= & \frac{1}{2}\left\|u_{t}(t)\right\|^{2}+\frac{1}{2}\left(1-\int_{0}^{t} g(s) d s\right)\|\Delta u(t)\|^{2}+\frac{1}{2}(g \circ \Delta u) \\
& +\frac{\xi}{2} \int_{t-\tau(t)}^{t} e^{-\lambda(t-s)}\left\|\nabla u_{t}(s)\right\|^{2} d s,
\end{aligned}
$$

where $\xi>0$ is a constant to be determined later, the constant $\lambda>0$, as below, has been introduced in [13],

$$
\lambda<\frac{1}{\tau_{1}}\left|\log \frac{\left|\mu_{2}\right|}{\sqrt{1-d}}\right|
$$

and we have

$$
(g \circ v)(t)=\int_{0}^{t} g(t-s)\|v(t)-v(s)\|^{2} d s
$$

Our main stability result is the following theorem.

Theorem 2.2 Assume the assumptions (2.1)-(2.4) hold. Let $\left|\mu_{2}\right|<\sqrt{1-d} \mu_{1}$. Let $\left(u, u_{t}\right)$ be the weak solutions of problem (1.1)-(1.3) with the initial data $\left(u_{0}, u_{1}\right) \in\left(H_{0}^{1}(\Omega) \times L^{2}(\Omega)\right)$, $f_{0} \in H^{1}(\Omega \times(-\tau(0), 0))$. Then there exist two constants $\beta>0$ and $\gamma>0$ such that the energy $E(t)$ satisfies

$$
E(t) \leq \beta \exp \left(-\gamma \int_{0}^{t} \zeta(s) d s\right), \text { for all } t \geq 0
$$




\section{Proof of Theorem 2.2}

In this section, we shall study the general decay of energy to problem (1.1)-(1.3) to prove Theorem 2.2. For this purpose, we need the following technical lemmas.

Lemma 3.1 Under the assumptions of Theorem 2.2, the energy functional $E(t)$ satisfies, for any $t \geq 0$,

$$
\begin{aligned}
E^{\prime}(t) \leq & \frac{1}{2}\left(g^{\prime} \circ \nabla u\right)(t)-\frac{1}{2} g(t)\|\nabla u(t)\|^{2}+\left(\frac{\xi}{2}-\mu_{1}+\frac{\left|\mu_{2}\right|}{2 \sqrt{1-d}}\right)\left\|\nabla u_{t}(t)\right\|^{2} \\
& +\left[\frac{\left|\mu_{2}\right|}{2} \sqrt{1-d}-\frac{\xi}{2}(1-d) e^{-\lambda \tau_{1}}\right]\left\|\nabla u_{t}(t-\tau(t))\right\|^{2} \\
& -\frac{\lambda \xi}{2} \int_{t-\tau(t)}^{t} e^{-\lambda(t-s)}\left\|\nabla u_{t}(s)\right\|^{2} d s .
\end{aligned}
$$

Proof Differentiating (2.5) and using (1.1), (2.3)-(2.4) and integration by parts, we have

$$
\begin{aligned}
E(t)= & \int_{\Omega} u_{t} u_{t t} d x-\frac{1}{2} g(t)\|\nabla u\|^{2}+\left(1-\int_{0}^{t} g(s) d s\right) \int_{\Omega} \nabla u \cdot \nabla u_{t} d x+\frac{1}{2}\left(g^{\prime} \circ \nabla u\right) \\
& +\int_{0}^{t} g(t-s) d s \cdot \int_{\Omega} \nabla u \cdot \nabla u_{t} d x-\int_{\Omega} \nabla u_{t}(t) \cdot \int_{0}^{t} g(t-s) \nabla u(s) d s d x \\
& +\frac{\xi}{2}\left\|\nabla u_{t}\right\|^{2}-\frac{\xi}{2} e^{-\lambda \tau(t)}\left(1-\tau^{\prime}(t)\right)\left\|\nabla u_{t}(t-\tau(t))\right\|^{2} \\
& -\frac{\lambda \xi}{2} \int_{t-\tau(t)}^{t} e^{-\lambda(t-s)}\left\|\nabla u_{t}(s)\right\|^{2} d s \\
\leq & -\mu_{1}\left\|\nabla u_{t}\right\|^{2}-\frac{1}{2} g(t)\|\nabla u\|^{2}+\frac{1}{2}\left(g^{\prime} \circ \nabla u\right)+\frac{\xi}{2}\left\|\nabla u_{t}\right\|^{2} \\
& -\mu_{2} \int_{\Omega} \nabla u_{t} \cdot \nabla u_{t}(t-\tau(t)) d x-\frac{\xi}{2}(1-d) e^{-\lambda \tau_{1}}\left\|\nabla u_{t}(t-\tau(t))\right\|^{2} \\
& -\frac{\lambda \xi}{2} \int_{t-\tau(t)}^{t} e^{-\lambda(t-s)}\left\|\nabla u_{t}(s)\right\|^{2} d s .
\end{aligned}
$$

It follows from Young's inequality that

$$
-\mu_{2} \int_{\Omega} \nabla u_{t} \cdot \nabla u_{t}(t-\tau(t)) d x \leq \frac{\left|\mu_{2}\right|}{2 \sqrt{1-d}}\left\|\nabla u_{t}(t)\right\|^{2}+\frac{\left|\mu_{2}\right|}{2} \sqrt{1-d}\left\|\nabla u_{t}(t-\tau(t))\right\|^{2},
$$

which, together with (3.2) gives us (3.1). The proof is done.

Lemma 3.2 Under the assumptions of Theorem 2.2, then the functional $\phi(t)$ defined as

$$
\phi(t)=\int_{\Omega} u(t) u_{t}(t) d x
$$

satisfies the requirement that there exist positive constants $c_{1}, c_{2}$ and $c_{3}$ such that, for any $t \geq 0$,

$$
\phi^{\prime}(t) \leq-\frac{l}{2}\|\nabla u(t)\|^{2}+c_{1}\left\|\nabla u_{t}(t)\right\|^{2}+c_{2}\left\|\nabla u_{t}(t-\tau(t))\right\|^{2}+c_{3}(g \circ \nabla u)(t) .
$$


Proof By using (1.1), we can get

$$
\begin{aligned}
& \phi^{\prime}(t)=\int_{\Omega} u_{t t} u d x+\left\|u_{t}\right\|^{2} \\
& =\left\|u_{t}\right\|^{2}+\int_{\Omega} u(t) \cdot\left(\Delta u-\int_{0}^{t} g(t-s) \Delta u(s) d s+\mu_{1} \Delta u_{t}+\mu_{2} \Delta u_{t}(t-\tau(t))\right) d x \\
& =\left\|u_{t}\right\|^{2}-\|\nabla u\|^{2}+\int_{\Omega} \nabla u(t) \cdot \int_{0}^{t} g(t-s)(\nabla u(s)-\nabla u(t)) d s d x \\
& +\int_{0}^{t} g(s) d s \cdot\|\nabla u\|^{2}+\mu_{1} \int_{\Omega} u \cdot \Delta u_{t} d x-\mu_{2} \int_{\Omega} \nabla u \cdot \nabla u_{t}(t-\tau(t)) d x \\
& \leq\left\|u_{t}\right\|^{2}-l\|\nabla u\|^{2}+\underbrace{\int_{\Omega} \nabla u(t) \cdot \int_{0}^{t} g(t-s)(\nabla u(s)-\nabla u(t)) d s d x}_{:=I_{1}} \\
& +\underbrace{\mu_{1} \int_{\Omega} u \cdot \Delta u_{t} d x}_{:=I_{2}}-\underbrace{\mu_{2} \int_{\Omega} \nabla u \cdot \nabla u_{t}(t-\tau(t)) d x}_{:=I_{3}} .
\end{aligned}
$$

By using Young's inequality and Hölder's inequality, we shall see below, for any $\delta>0$,

$$
\begin{aligned}
I_{1} & \leq \delta\|\nabla u\|^{2}+\frac{1}{4 \delta} \int_{\Omega}\left(\int_{0}^{t} g(t-s)(\nabla u(s)-\nabla u(t)) d s\right)^{2} d x \\
& \leq \delta\|\nabla u\|^{2}+\frac{1}{4 \delta} \int_{0}^{t} g(s) d s(g \circ \nabla u)(t) \\
& \leq \delta\|\nabla u\|^{2}+\frac{1-l}{4 \delta}(g \circ \nabla u)(t), \\
I_{2} & \leq \delta\|\nabla u\|^{2}+\frac{\mu_{1}^{2}}{4 \delta}\left\|\nabla u_{t}\right\|^{2}, \\
I_{3} & \leq \delta\|\nabla u\|^{2}+\frac{\mu_{2}^{2}}{4 \delta}\left\|\nabla u_{t}(t-\tau(t))\right\|^{2},
\end{aligned}
$$

which, together with (3.5)-(3.7), implies, for any $\delta>0$,

$$
\phi^{\prime}(t) \leq\left\|u_{t}\right\|^{2}-(l-3 \delta)\|\nabla u\|^{2}+\frac{\mu_{1}^{2}}{4 \delta}\left\|\nabla u_{t}\right\|^{2}+\frac{\mu_{2}^{2}}{4 \delta}\left\|\nabla u_{t}(t-\tau(t))\right\|^{2}+\frac{1-l}{4 \delta}(g \circ \nabla u)(t) .
$$

Now taking $\delta>0$ small enough such that

$$
l-3 \delta>\frac{l}{2}
$$

we can get the desired estimate (3.4) with

$$
c_{1}=\frac{1}{\lambda_{1}}+\frac{\mu_{1}^{2}}{4 \delta}, \quad c_{2}=\frac{\mu_{2}^{2}}{4 \delta}, \quad c_{3}=\frac{1-l}{4 \delta},
$$

hereafter the positive constant $\lambda_{1}$ represents the Poincaré's constant, i.e., $\lambda_{1}\|u\|^{2} \leq\|\nabla u\|$ for $u \in H_{0}^{1}(\Omega)$. The proof is hence complete. 
Lemma 3.3 We define the functional $\psi(t)$ as

$$
\psi(t)=-\int_{\Omega} u_{t}(t) \cdot \int_{0}^{t} g(t-s)(u(t)-u(s)) d s d x
$$

Under the assumptions of Theorem 2.2, the functional $\psi(t)$ satisfies, for any $\delta>0$,

$$
\begin{aligned}
\psi^{\prime}(t) \leq & -\left(\int_{0}^{t} g(s) d s-\delta\right)\left\|u_{t}\right\|^{2}+\delta\left\|\nabla u_{t}(t)\right\|^{2}+\delta\left\|\nabla u_{t}(t-\tau(t))\right\|^{2} \\
& +c_{4}(g \circ \Delta u)(t)-c_{5}\left(g^{\prime} \circ \Delta u\right)(t),
\end{aligned}
$$

where $c_{4}$ and $c_{5}$ are positive constants.

Proof It follows from (1.1) and integration by parts that

$$
\begin{aligned}
\psi^{\prime}(t)= & -\int_{\Omega} u_{t t} \cdot \int_{0}^{t} g(t-s)(u(t)-u(s)) d s d x \\
& -\int_{\Omega} u_{t}\left[u_{t} \int_{0}^{t} g(t-s) d s+\int_{0}^{t} g^{\prime}(t-s)(u(t)-u(s)) d s\right] d x \\
= & \int_{\Omega}\left(-\Delta u+\int_{0}^{t} g(t-s) \Delta u(s) d s-\nabla u_{t}-\mu_{2} \Delta u_{t}(t-\tau(t))\right) \\
& \times \int_{0}^{t} g(t-s)(u(t)-u(s)) d s d x-\int_{0}^{t} g(s) d s\left\|u_{t}\right\|^{2} \\
& -\int_{\Omega} u_{t} \int_{0}^{t} g^{\prime}(t-s)(u(t)-u(s)) d s d x \\
= & \left(1-\int_{0}^{t} g(s) d s\right) \int_{\Omega} \nabla u(t) \cdot \int_{0}^{t} g(t-s)(\nabla u(t)-\nabla u(s)) d s d x \\
& +\int_{\Omega}\left(\int_{0}^{t} g(t-s)(\nabla u(s)-\nabla u(t)) d s\right)^{2} d x-\int_{0}^{t} g(s) d s\left\|u_{t}\right\|^{2} \\
& -\int_{\Omega} u_{t} \int_{0}^{t} g^{\prime}(t-s)(u(t)-u(s)) d s d x \\
& +\mu_{1} \int_{\Omega} \nabla u_{t}(t) \cdot \int_{0}^{t} g(t-s)(\nabla u(t)-\nabla u(s)) d s d x \\
& +\mu_{2} \int_{\Omega} \nabla u_{t}(t-\tau(t)) \cdot \int_{0}^{t} g(t-s)(\nabla u(t)-\nabla u(s)) d s d x .
\end{aligned}
$$

By using Hölder's inequality, Young's inequality and the Poincaré inequality, we can obtain, for any $\delta>0$,

$$
\begin{aligned}
& \mu_{2} \int_{\Omega} \nabla u \int_{0}^{t} g(t-s)(\nabla u(t)-\nabla u(s)) d s d x \leq \delta\left\|\nabla u_{t}\right\|^{2}+\frac{\mu_{1}^{2}(1-l)}{4 \delta}(g \circ \nabla u)(t), \\
& \mu_{2} \int_{\Omega} \nabla u_{t}(t-\tau(t)) \cdot \int_{0}^{t} g(t-s)(\nabla u(t)-\nabla u(s)) d s d x \\
& \quad \leq \delta\left\|\nabla u_{t}(t-\tau(t))\right\|^{2}+\frac{\mu_{2}^{2}(1-l)}{4 \delta}(g \circ \nabla u)(t),
\end{aligned}
$$




$$
\begin{aligned}
& -\int_{\Omega} u_{t} \int_{0}^{t} g^{\prime}(t-s)(u(t)-u(s)) d s d x \\
& \quad \leq \delta\left\|u_{t}\right\|^{2}+\frac{1}{4 \delta}\left(\int_{0}^{t}\left(-g^{\prime}(t-s)\right)\|u(t)-u(s)\| d s\right)^{2} \\
& \quad \leq \delta\left\|u_{t}\right\|^{2}-\frac{C g(0)}{4 \delta \lambda_{1}}\left(g^{\prime} \circ \Delta u\right)(t),
\end{aligned}
$$

and

$$
\int_{\Omega}\left(\int_{0}^{t} g(t-s)(\nabla u(s)-\nabla u(t)) d s\right)^{2} d x \leq \frac{1-l}{\lambda_{1}}(g \circ \nabla u)(t),
$$

which, combined (3.12)-(3.14) with (3.11), gives us (3.10). The proof is therefore complete.

Now we define the Lyapunov functional $F(t)$ by

$$
F(t):=E(t)+\varepsilon_{1} \phi(t)+\varepsilon_{2} \psi(t)
$$

where $\varepsilon_{1}$ and $\varepsilon_{2}$ are positive constants to be taken later. First we know that, for $\varepsilon_{1}>0$ and $\varepsilon_{2}>0$ small enough, there exist two positive constants $\beta_{1}$ and $\beta_{2}$ such that, for any $t>0$,

$$
\beta_{1} E(t) \leq F(t) \leq \beta_{2} E(t) .
$$

Proof of Theorem 2.2 For any $t_{0}>0$, we get, for any $t \geq t_{0}$,

$$
\int_{0}^{t} g(s) d s \geq \int_{0}^{t_{0}} g(s) d s:=g_{0}
$$

It follows from (3.1), (3.4) and (3.10) that, for any $t \geq t_{0}$,

$$
\begin{aligned}
F^{\prime}(t) \leq & -\left(\int_{0}^{t} g(s) d s-\delta\right) \varepsilon_{2}\left\|u_{t}(t)\right\|^{2}-\left(\frac{l}{2} \varepsilon_{1}-\delta \varepsilon_{2}\right)\|\nabla u(t)\|^{2} \\
& +\left(\frac{\xi}{2}-\mu_{1}+\frac{\left|\mu_{2}\right|}{2 \sqrt{1-d}}+c_{1} \varepsilon_{1}+\delta \varepsilon_{2}\right)\left\|\nabla u_{t}(t)\right\|^{2} \\
& +\left[\frac{\left|\mu_{2}\right|}{2} \sqrt{1-d}-\frac{\xi}{2}(1-d) e^{-\lambda \tau_{1}}+c_{2} \varepsilon_{1}+\delta \varepsilon_{2}\right]\left\|\nabla u_{t}(t-\tau(t))\right\|^{2} \\
& +\left(\frac{1}{2}-c_{5} \varepsilon_{2}\right)\left(g^{\prime} \circ \nabla u\right)(t)+\left(c_{3} \varepsilon_{1}+c_{4} \varepsilon_{2}\right)(g \circ \nabla u)(t) \\
& -\frac{\lambda \xi}{2} \int_{t-\tau(t)}^{t} e^{-\lambda(t-s)}\left\|\nabla u_{t}(s)\right\|^{2} d s .
\end{aligned}
$$

Obviously, $e^{\lambda \tau_{1}} \rightarrow 1$ as $\lambda \rightarrow 0$. Because of the continuity of the set of real numbers, we pick $\lambda>0$ small enough such that there exists a positive constant $\xi$ such that

$$
\frac{e^{\lambda \tau_{1}}\left|\mu_{2}\right|}{\sqrt{1-d}}<\xi<\mu_{1}
$$


which implies

$$
\frac{\left|\mu_{2}\right|}{2 \sqrt{1-d}}-\mu_{1}+\frac{\xi}{2}<0 \quad \text { and } \quad \frac{\left|\mu_{2}\right|}{2} \sqrt{1-d}-\frac{\xi}{2 e^{\lambda \tau_{1}}}(1-d)<0 \text {. }
$$

It follows from (3.18) and (3.19) that the energy functional (2.5) is nonincreasing.

Now we take $\delta>0$ small enough such that, for $t \geq t_{0}$,

$$
\int_{0}^{t} g(s) d s-\delta \geq \frac{1}{2} g_{0}
$$

At this point, for any fixed $\delta>0$, we choose $\varepsilon_{2}>0$ so small that (3.16) holds, and further

$$
\varepsilon_{2}<\min \left\{\frac{1}{4 c_{5}}, \frac{\mu_{1}}{2 \delta}-\frac{\xi}{4 \delta}-\frac{\left|\mu_{2}\right|}{4 \delta \sqrt{1-d}}, \frac{\xi}{4 \delta}(1-d) e^{-\lambda \tau_{1}}-\frac{\left|\mu_{2}\right|}{4 \delta} \sqrt{1-d}\right\},
$$

which yields

$$
\frac{1}{2}-c_{5} \varepsilon_{2}>\frac{1}{4}, \quad \frac{\xi}{2}-\mu_{1}+\frac{\left|\mu_{2}\right|}{2 \sqrt{1-d}}+\delta \varepsilon_{2}<\frac{\xi}{4}-\frac{\mu_{1}}{2}+\frac{\left|\mu_{2}\right|}{4 \sqrt{1-d}}
$$

and

$$
\frac{\left|\mu_{2}\right|}{2} \sqrt{1-d}-\frac{\xi}{2}(1-d) e^{-\lambda \tau_{1}}+\delta \varepsilon_{2}<\frac{\left|\mu_{2}\right|}{4} \sqrt{1-d}-\frac{\xi}{4}(1-d) e^{-\lambda \tau_{1}} .
$$

In the sequel, for any fixed $\delta>0$ and $\varepsilon_{2}>0$, we take $\varepsilon_{1}>0$ small so that (3.16) holds, and further

$$
\frac{4 \delta \varepsilon_{2}}{l}<\varepsilon_{1}<\min \left\{\frac{\mu_{1}}{4 c_{1}}-\frac{\xi}{8 c_{1}}-\frac{\left|\mu_{2}\right|}{8 c_{1} \sqrt{1-d}}, \frac{\xi}{8 c_{2}}(1-d) e^{-\lambda \tau_{1}}-\frac{\left|\mu_{2}\right|}{8 c_{2}} \sqrt{1-d}\right\},
$$

which gives us

$$
\frac{l}{2} \varepsilon_{1}-\delta \varepsilon_{2}>\frac{l}{4} \varepsilon_{2}, \quad \frac{\xi}{4}-\frac{\mu_{1}}{2}+\frac{\left|\mu_{2}\right|}{4 \sqrt{1-d}}+c_{1} \varepsilon_{1}<\frac{\xi}{8}-\frac{\mu_{1}}{4}+\frac{\left|\mu_{2}\right|}{8 \sqrt{1-d}}
$$

and

$$
\frac{\left|\mu_{2}\right|}{4} \sqrt{1-d}-\frac{\xi}{4}(1-d) e^{-\lambda \tau_{1}}+c_{2} \varepsilon_{1}<\frac{\left|\mu_{2}\right|}{8} \sqrt{1-d}-\frac{\xi}{8}(1-d) e^{-\lambda \tau_{1}} .
$$

From the above we know that, for positive constants $\alpha_{1}$ and $\alpha_{2}$,

$$
F^{\prime}(t) \leq-\alpha_{1} E(t)+\alpha_{2}(g \circ \nabla u)(t), \quad \forall t \geq t_{0} .
$$

Multiplying (3.20) by $\zeta(t)$ and using (2.2), we can get, for any $t \geq t_{0}$,

$$
\begin{aligned}
\zeta(t) F^{\prime}(t) & \leq-\alpha_{1} \zeta(t) E(t)+\alpha_{2} \zeta(t)(g \circ \Delta u)(t) \\
& \leq-\alpha_{1} \zeta(t) E(t)-\alpha_{2}\left(g^{\prime} \circ \Delta u\right)(t) \\
& \leq-\alpha_{1} \zeta(t) E(t)-\alpha_{3} E^{\prime}(t)
\end{aligned}
$$


where $\alpha_{3}>0$. Denote $\mathcal{E}(t)=\zeta(t) F(t)+\alpha_{3} E(t)$, then it is easy to see that $\mathcal{E}(t)$ is equivalent to the energy $E(t)$, i.e., there exist two positive constants $\beta_{3}$ and $\beta_{4}$ such that

$$
\beta_{3} E(t) \leq \mathcal{E}(t) \leq \beta_{4} E(t)
$$

Thus we can infer that, for any $t \geq t_{0}$,

$$
\mathcal{E}^{\prime}(t) \leq-\frac{\alpha_{1}}{\beta_{4}} \zeta(t) \mathcal{E}(t)
$$

which, integrating over $\left(t_{0}, t\right)$ with respect to $t$, yields, for any $t \geq t_{0}$,

$$
\mathcal{E}(t) \leq \mathcal{E}\left(t_{0}\right) \exp \left(-\frac{\alpha_{1}}{\beta_{4}} \int_{t_{0}}^{t} \zeta(s) d s\right)
$$

Therefore (2.6) follows from (3.23) by renaming the constants, and by the continuity and boundedness of $E(t)$ and $\zeta(t)$. The proof is hence complete.

Remark 3.1 If taking $\zeta(t)=\gamma$ and $\zeta(t)=\gamma(1+t)^{-1}$, and $\gamma$ a positive constant, we can obtain the exponential decay and polynomial decay of problem (1.1)-(1.3), respectively. Thus the exponential decay and polynomial decay is a particular case of (2.6). We also find some other examples to illustrate several rates of energy decay; see, for example, [25, 32].

Competing interests

The author declares that he has no competing interests.

\section{Publisher's Note}

Springer Nature remains neutral with regard to jurisdictional claims in published maps and institutional affiliations.

Received: 27 February 2017 Accepted: 30 March 2017 Published online: 20 April 2017

\section{References}

1. Nicaise, S, Pignotti, C: Stability and instability results of the wave equation with a delay term in the boundary or internal feedbacks. SIAM J. Control Optim. 45, 1561-1585 (2006)

2. Kirane, M, Said-Houari, B: Existence and asymptotic stability of a viscoelastic wave equation with a delay. Z. Angew. Math. Phys. 62, 1065-1082 (2011)

3. Liu, WJ: General decay of the solution for viscoelastic wave equation with a time-varying delay term in the internal feedback. J. Math. Phys. 54, 043504 (2013)

4. Dai, Q, Yang, Z: Global existence and exponential decay of the solution for a viscoelastic wave equation with a delay. Z. Angew. Math. Phys. 65, 885-903 (2014)

5. Benaissa, A, Benguessoum, A, Messaoudi, SA: Global existence and energy decay of solutions to a viscoelastic wave equation with a delay term in the nonlinear internal feedback. Int. J. Dyn. Syst. Differ. Equ. 5(1), 1-26 (2014)

6. Liu, G, Zhang, H: Well-posedness for a class of wave equation with past history and a delay. Z. Angew. Math. Phys. 67, 6 (2016). doi:10.1007/s00033-015-0593-Z

7. Kafini, M, Messaoudi, SA, Nicaise, S: A blow-up result in a nonlinear abstract evolution system with delay. NoDEA Nonlinear Differ. Equ. Appl. (2016). doi:10.1007/s00030-016-0371-4

8. Alabau-Boussouira, F, Nicaise, S, Pignotti, C: Exponential stability of the wave equation with memory and time delay. In: New Prospects in Direct, Inverse and Control Problems for Evolution Equations. Springer INdAM Series, vol. 10, pp. 1-22 (2014)

9. Benaissa, A, Benguessoum, A, Messaoudi, SA: Energy decay of solutions for a wave equation with a constant weak delay and a weak internal feedback. Electron. J. Qual. Theory Differ. Equ. 2014, 11 (2014)

10. Datko, R, Lagnese, J, Polis, MP: An example on the effect of time delays in boundary feedback stabilization of wave equations. SIAM J. Control Optim. 24, 152-156 (1986)

11. Liu, WJ: General decay rate estimate for the energy of a weak viscoelastic equation with an internal time-varying delay term. Taiwan. J. Math. 17, 2101-2115 (2013)

12. Nicaise, S, Pignotti, C: Exponential stability of abstract evolution equations with time delay. J. Evol. Equ. 15, 107-129 (2015)

13. Nicaise, S, Pignotti, C: Interior feedback stabilization of wave equations with time dependent delay. Electron. J. Differ. Equ. 2011, 41 (2011) 
14. Nicaise, S, Pignotti, C: Stabilization of the wave equation with boundary or internal distributed delay. Differ. Integral Equ. 21, 935-958 (2008)

15. Nicaise, S, Valein, J, Fridman, E: Stabilization of the heat and the wave equations with boundary time-varying delays. Discrete Contin. Dyn. Syst., Ser. S 2, 559-581 (2009)

16. Nicaise, S, Valein, J: Stabilization of second order evolution equations with unbounded feedback with delay. ESAIM Control Optim. Calc. Var. 16, 420-456 (2010)

17. $\mathrm{Xu}, \mathrm{G}$, Yung, $\mathrm{S}, \mathrm{Li}, \mathrm{L}:$ Stabilization of wave systems with input delay in the boundary control. ESAIM Control Optim. Calc. Var. 12, 770-785 (2006)

18. Messaoudi, SA, Fareh, A, Doudi, N: Well posedness and exponential stability in a wave equation with a strong damping and a strong delay. J. Math. Phys. 57, 111501 (2016)

19. Berrimi, S, Messaoudi, SA: Existence and decay of solutions of a viscoelastic equation with a nonlinear source. Nonlinear Anal. 64, 2314-2331 (2006)

20. Cavalcanti, MM, Domingos Cavalcanti, VN, Ferreira, J: Existence and uniform decay of nonlinear viscoelastic equation with strong damping. Math. Methods Appl. Sci. 24, 1043-1053 (2001)

21. Cavalcanti, MM, Domingos Cavalcanti, VN, Soriano, JA: Exponential decay for the solution of semilinear viscoelastic wave equation with localized damping. Electron. J. Differ. Equ. 2002, 44 (2002)

22. Han, X, Wang, M: General decay of energy for a viscoelastic equation with nonlinear damping. Math. Methods Appl. Sci. 32, 346-358 (2009)

23. Han, X, Wang, M: Global existence and uniform decay for a nonlinear viscoelastic equation with damping. Nonlinear Anal. TMA 70, 3090-3098 (2009)

24. Liu, WJ: General decay rate estimates for a viscoelastic equation with weakly nonlinear time-dependent dissipation and source terms. J. Math. Phys. 50, 113506 (2009)

25. Messaoudi, SA: General decay of solutions of a weak viscoelastic equation. Arab. J. Sci. Eng. 36, 1569-1579 (2011)

26. Messaoudi, SA: General decay of solution energy in a viscoelastic equation with a nonlinear source. Nonlinear Anal. 69, 2589-2598 (2008)

27. Messaoudi, SA: General decay of solutions of a viscoelastic equation. J. Math. Anal. Appl. 341, 1457-1467 (2008)

28. Messaoudi, SA, Tartar, N-E: Exponential and polynomial decay for a quasilinear viscoelastic equation. Nonlinear Anal. 68, 785-793 (2008)

29. Messaoudi, SA, Tatar, N-E: Global existence and uniform stability of solutions for a quasilinear viscoelastic problem. Math. Methods Appl. Sci. 30, 665-680 (2007)

30. Messaoudi, SA, Tartar, N-E: Global existence and asymptotic behavior for a nonlinear viscoelastic equation. Math. Sci. Res. J. 7(4), 136-149 (2003)

31. Messaoudi, SA, Al-Gharabli, MM: A general decay result of a viscoelastic equation with past history and boundary feedback. Z. Angew. Math. Phys. 66, 1519-1528 (2015)

32. Tatar, N-E: Arbitrary decays in linear viscoelasticity. J. Math. Phys. 52, 013502 (2011)

\section{Submit your manuscript to a SpringerOpen ${ }^{\circ}$ journal and benefit from:}

- Convenient online submission

Rigorous peer review

- Immediate publication on acceptance

- Open access: articles freely available online

- High visibility within the field

- Retaining the copyright to your article 